\title{
Fitting corrections to an RNA force field using experimental data
}

Andrea Cesari, Sandro Bottaro, Kresten Lindorff-Larsen, Pavel Banas, Jiri

Sponer, and Giovanni Bussi*

E-mail: bussi@sissa.it 


\section{Supplementary methods}

Table S1: Systems composing the training set in the the force-field refinement procedure. Employed data type for each system are reported (e.g. NOE) with the relative forward model used to back-calculate them from simulations data. In the case of NOE data, index $i$ runs over the proton pairs for which NOE data are available. NOE and ${ }^{3} J$ couplings experimental data were taken from Refs. ${ }^{1-3}$ In the case of ${ }^{3} J$ couplings, the index $i$ runs over all torsions for which scalar couplings are available. In the case of tetraloops, index $i$ refers to the system for which the folded fraction is computed (i.e. cCGAGAgg or ccUUCGgg). Parameters $\omega_{\mathrm{NOE}}=1100 \mathbf{n m}^{-2}, \omega_{\mathbf{3} \mathbf{J}}=2 \mathbf{H z}^{-2}$, and $\omega_{\mathbf{T L}}=20$ represent the weights of each set of data in the error function used in the fitting procedure and have been heuristically chosen in order to reproduce the expected experimental error of a given experiment type.

\begin{tabular}{|c|c|c|c|}
\hline System & Forward Model & Constraint & Error Function \\
\hline \multirow{4}{*}{$\begin{array}{l}\text { AAAA } \\
\text { CCCC } \\
\text { UUUU } \\
\text { GACC }\end{array}$} & \multicolumn{3}{|c|}{ NOE } \\
\hline & \multirow[t]{2}{*}{$\left\langle O_{i}\right\rangle=\left\langle\frac{1}{d_{i}^{6}}\right\rangle^{-\frac{1}{6}}$} & $d_{i, \text { min }}^{e x p} \leq\left\langle O_{i}\right\rangle \leq d_{i, \text { max }}^{e x p}$ & $\begin{array}{l}\omega_{\mathrm{NOE}} \max \left(\left\langle\frac{1}{d^{6}}\right\rangle^{-1 / 6}-d_{i, \max }^{\exp }, 0\right)^{2}+ \\
+\omega_{\mathrm{NOE}} \max \left(d_{i, \min }^{\exp }-\left\langle\frac{1}{d_{i}^{6}}\right\rangle^{-1 / 6}, 0\right)^{2}\end{array}$ \\
\hline & & \multicolumn{2}{|l|}{${ }^{3} \mathrm{~J}$ Couplings } \\
\hline & $\left\langle O_{i}\right\rangle=A \cos (2 x)+B \cos (x)+C=\left\langle{ }^{3} J_{i}\right\rangle$ & $\left\langle O_{i}\right\rangle=O_{i}^{\text {exp }}=\left\langle{ }^{3} J^{\exp }\right\rangle$ & $\omega_{3 J}\left(\left\langle\left\langle^{3} J_{i}\right\rangle-{ }^{3} J_{i}^{e x p}\right)^{2}\right.$ \\
\hline \multirow[b]{2}{*}{$\begin{array}{l}\text { ccGAGAgg } \\
\text { ccUUCGg }\end{array}$} & \multicolumn{3}{|c|}{ Native Fraction } \\
\hline & $\begin{array}{c}\left\langle O_{i}\right\rangle=\left\langle p_{f}^{i}(t)\right\rangle ; \\
p_{f}^{i}(t)= \begin{cases}1 & \text { eRMSD } \leq 0.8 \\
0 & \text { otherwise }\end{cases} \end{array}$ & $\left\langle p_{f}^{i}(t)\right\rangle \geq 0.5$ & $\omega_{\mathrm{TL}} \max \left(0, \log (0.5)-\log \left(\left\langle p_{f}^{i}(t)\right\rangle\right)\right)^{2}$ \\
\hline
\end{tabular}




\section{Supplementary results for tetraloops}

We here report supplementary analysis of the tetraloop trajectories.

Table S2: Numerical population of the native state for both the investigated tetraloops using the four analyzed ensembles. Results in the second and fourth columns are obtained by defining as folded all the states with eRMSD from native smaller than 0.8, computing eRMSD with an augmented distance cutoff equal to 3.2. These populations are computed consistently with those used in the training procedure. Results in the third and fifth columns are obtained by defining as folded all the states with eRMSD from native smaller than $\mathbf{0 . 7}$, computing eRMSD with the standard distance cutoff equal to 2.4. This latter criterion has been used in Ref. ${ }^{4}$ and was shown to select only structures with a virtually identical annotation of all canonical and non-canonical base pairs and stacking interactions. Although the populations are quantitatively different, the trends are preserved.

\begin{tabular}{|l|cc|cc|}
\hline System & \multicolumn{2}{|c|}{ GAGA } & \multicolumn{2}{c|}{ UUCG } \\
\hline Metric & eRMSD $\leq 0.8$ & eRMSD $^{*} \leq 0.7$ & eRMSD $\leq 0.8$ & eRMSD* $^{*} 0.7$ \\
\hline Amber & 0.01840 & 0.02014 & 0.000018 & 0.00003 \\
Amber $_{\text {RW }}$ & 0.12713 & 0.14348 & 0.00129 & 0.00188 \\
Amber $_{\text {RWRES }}$ & 0.06647 & 0.05455 & 0.00082 & 0.00116 \\
DESRES & NA & NA & 0.00031 & 0.00036 \\
\hline
\end{tabular}



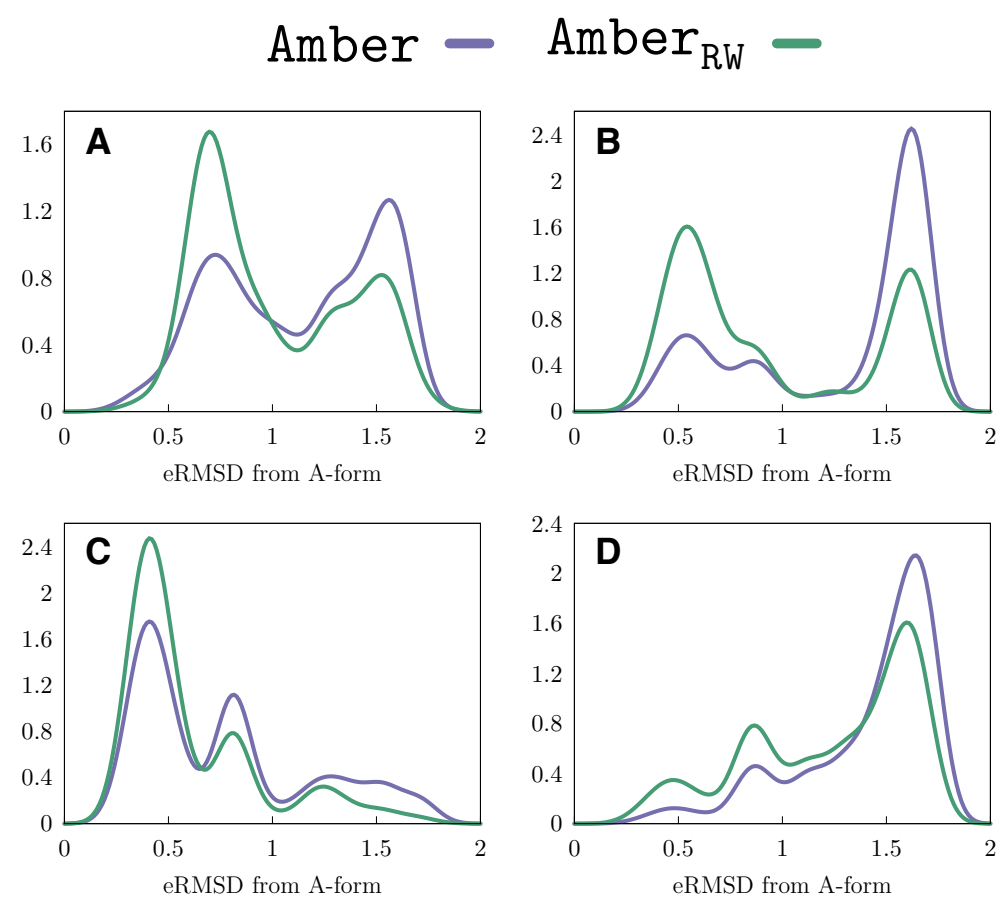

Figure S1: Histogram of eRMSD from a canonical A-form for the four tetranucleotides (panel A, AAAA; panel B, CCCC; panel C, GACC; panel D, UUUU). A-form corresponds to structures with a eRMSD less than $\approx 0.7$. For all systems, the population of $\mathrm{A}$ form is increased upon reweighting. 


\section{Effect of the regularization term}

We here report individual contributions to the error function with and without the regularization term.
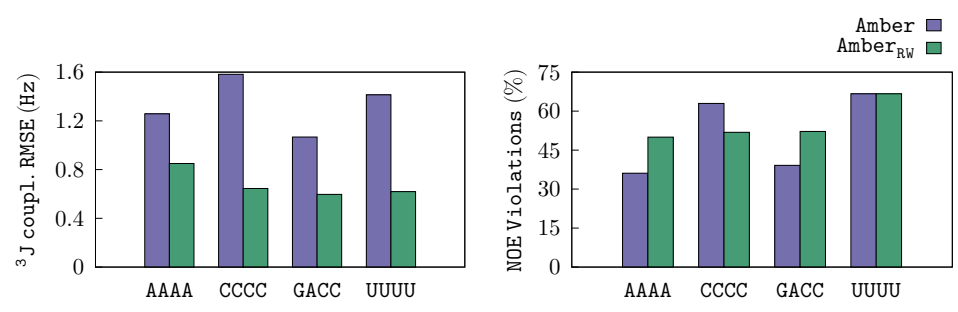

Figure S2: ${ }^{3} J$ scalar coupling RMSE and NOE violations for RNA tetranucleotides as obtained setting $\alpha=0$ (that is without any regularization term). For both RMSE and percentage of violations, the lower the better. Agreement with experiment is improved for ${ }^{3} \mathrm{~J}$ scalar coupling when compared with the fitting done with $\alpha=1500$ (main text). However, due to overfitting, agreement for NOE violations is worst.
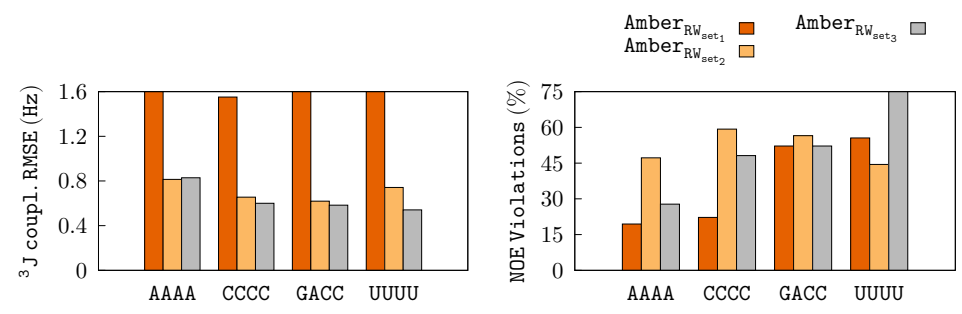

Figure S3: ${ }^{3} J$ scalar coupling RMSE and NOE violations for RNA tetranucleotides as obtained setting $\alpha=0$ (that is without any regularization term). For both RMSE and percentage of violations, the lower the better. Set 1 represents results obtained training the corrections on NOE violations and tetraloop stability. Set 2 represents results obtained training the corrections on ${ }^{3} J$ scalar coupling RMSE and tetraloop stability. Set 3 represents results obtained training the corrections on ${ }^{3} J$ scalar coupling RMSE NOE violations. The cross validation done on the left-out dataset (left panel, Set 1; right panel, Set 2) performs worst than the original Amber force field in most of the cases. 

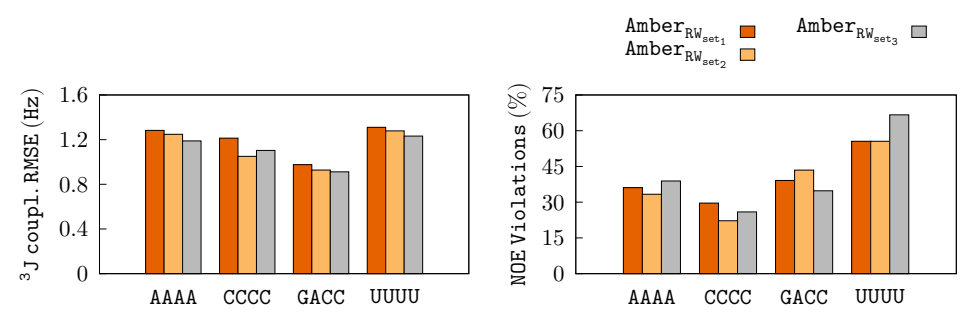

Figure S4: ${ }^{3} J$ scalar coupling RMSE and NOE violations for RNA tetranucleotides as obtained setting $\alpha=1500$. For both RMSE and percentage of violations, the lower the better. Set 1 represents results obtained training the corrections on NOE violations and tetraloop stability. Set 2 represents results obtained training the corrections on ${ }^{3} J$ scalar coupling RMSE and tetraloop stability. Set 3 represents results obtained training the corrections on ${ }^{3} J$ scalar coupling RMSE NOE violations. The performance is similar when the validated dataset is excluded (left panel, Set 1; right panel, Set 2) or included (all other cases).

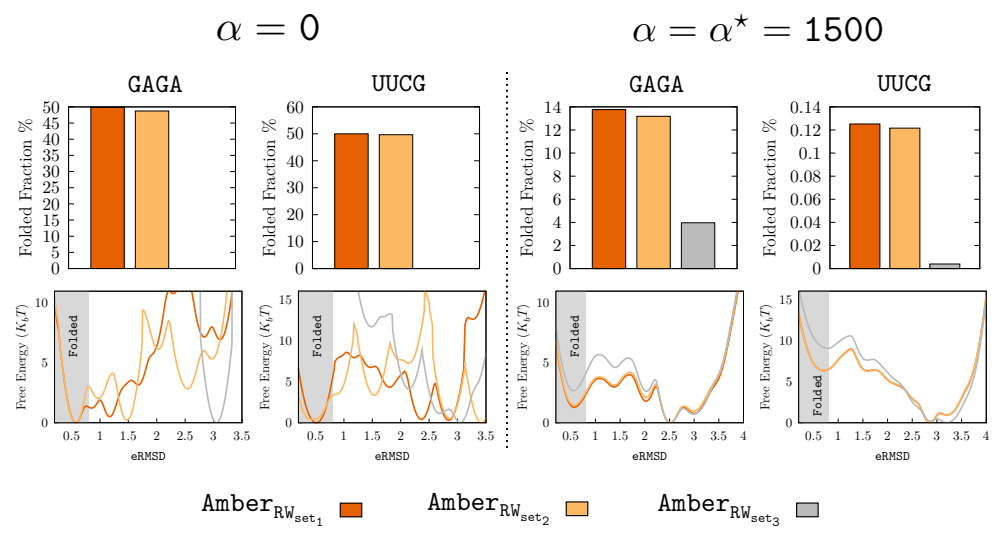

Figure S5: Fraction of folded structures (top) and free energy surface (bottom) with different force-field fitted against subsets of experimental data. Set 1 represents results obtained training the corrections on NOE violations and tetraloop stability. Set 2 represents results obtained training the corrections on ${ }^{3} J$ scalar coupling RMSE and tetraloop stability. Set 3 represents results obtained training the corrections on ${ }^{3} J$ scalar coupling RMSE NOE violations. When tetraloop data are not included (set 3) and no regularization term is used $(\alpha=0)$ the tetraloop stabilities are even lower than those obtained with the original Amber force field. On the other hand, when the optimal regularization term is used $(\alpha=1500)$ tetraloop stabilities are moderately improved with respect to the original Amber force field also when tetraloop data are not used in the fitting procedure. 
Table S3: We report here the values of the $\lambda_{\text {RWRES }}$ coefficients obtained with

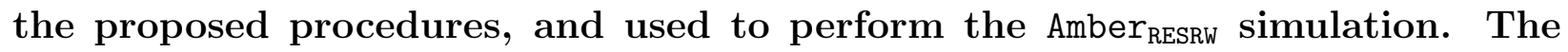
sum in the first column is meant to be taken on all the torsions of type $t$ present in the considered system. The quantity $k_{B} T \sqrt{6 \frac{\sum_{i=1}^{48} \lambda_{i}^{2}}{48}} \approx 0.68 \mathrm{~kJ} / \mathrm{mol}$ can be used as an indicator of the average corrections per torsion. A script that generates a PLUMED input to apply the corrections is available at http://github.com/bussilab/ff-fitting-tools. Notice that these dihedral terms should be applied on top of the correct starting force field, that for this work was the f99bsc0 $+\chi_{O L 3}$ Amber force field ${ }^{5-7}$ with corrections to van der Waals oxygen radii $^{8}$ and using the OPC water model. ${ }^{9}$ The force field is available at http://github.com/srnas/ff.

\begin{tabular}{|c|c|c|c|c|c|c|c|c|}
\hline$t$ & $\alpha$ & $\beta$ & $\gamma$ & $\delta$ & $\epsilon$ & $\zeta$ & $\chi_{\text {Pur }}$ & $\chi_{\text {Pyr }}$ \\
\hline \hline$\sum \cos (t)$ & 0.028743 & -0.118088 & 0.369240 & -0.068875 & -0.061779 & -0.083563 & -0.007880 & 0.067727 \\
\hline$\sum \cos (2 t)$ & -0.135683 & 0.199287 & -0.269494 & 0.035808 & 0.086680 & 0.091654 & -0.054599 & -0.116106 \\
\hline$\sum \cos (3 t)$ & 0.122662 & -0.064958 & -0.001949 & 0.109930 & 0.042434 & 0.061681 & 0.016977 & 0.037457 \\
\hline$\sum \sin (t)$ & 0.045960 & -0.085707 & 0.080645 & -0.011406 & 0.038967 & 0.220101 & -0.086418 & -0.014430 \\
\hline$\sum \sin (2 t)$ & 0.064641 & 0.008920 & 0.190771 & -0.109477 & 0.054064 & 0.150732 & -0.073151 & -0.046067 \\
\hline$\sum \sin (3 t)$ & 0.032150 & 0.016087 & 0.020993 & 0.051061 & -0.077758 & 0.015956 & 0.143994 & 0.140334 \\
\hline
\end{tabular}




\section{Weights validation using WHAM}

As a further check, we computed the native populations by combining the Amber and the Amber $_{\text {RESRW }}$ simulations using the weighted-histogram analysis method (WHAM) ${ }^{10}$ in order to take into account both the final bias ${ }^{11}$ introduced by the metadynamics potential and the difference between the two employed force fields. The resulting populations corresponding

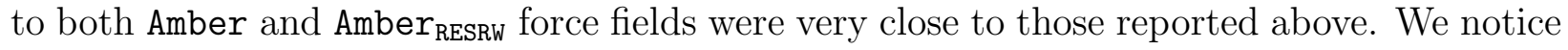
that, as suggested in Ref., ${ }^{12}$ one might reiterate the fitting procedure until satisfactory results are obtained. In our case, the $\lambda$ coefficients obtained by fitting on the WHAM

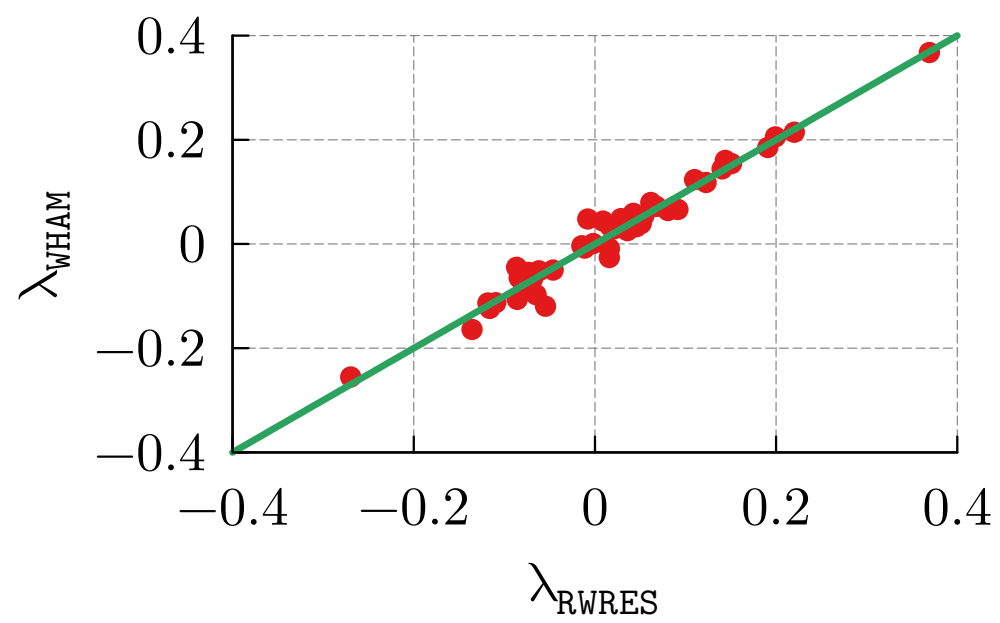

Figure S6: Comparison between the coefficients $\lambda_{\text {RWRES }}$ (reported in Tab. S3) and the $\lambda_{\text {WHAM }}$ coefficients obtained by performing the reweighting procedure on the WHAM-combined trajectory. Notice that with the formalism used in this paper the coefficients of $\lambda$ are unitless and they must be multiplied by the value of $k_{b} T$ when used for reweighting.

simulation using the same regularization parameter $\alpha=1500$ were very close to those used

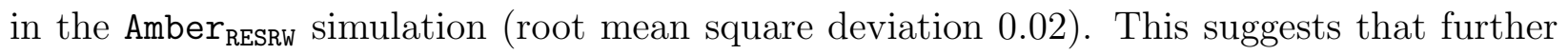
iterations are not required. Comparison between $\lambda$ coefficients and the values used to perform the Amber $r_{\text {RESRW }}$ are reported in Fig. S6. 


\section{References}

(1) Tubbs, J. D.; Condon, D. E.; Kennedy, S. D.; Hauser, M.; Bevilacqua, P. C.; Turner, D. H. The Nuclear Magnetic Resonance of CCCC RNA Reveals a Right-Handed Helix, and Revised Parameters for AMBER Force Field Torsions Improve Structural Predictions from Molecular Dynamics. Biochemistry 2013, 52, 996-1010.

(2) Condon, D. E.; Kennedy, S. D.; Mort, B. C.; Kierzek, R.; Yildirim, I.; Turner, D. H. Stacking in RNA: NMR of Four Tetramers Benchmark Molecular Dynamics. J. Chem. Theory Comput. 2015, 11, 2729-2742.

(3) Bottaro, S.; Bussi, G.; Kennedy, S. D.; Turner, D. H.; Lindorff-Larsen, K. Conformational ensembles of RNA oligonucleotides from integrating NMR and molecular simulations. Science Advances 2018, 4, eaar8521.

(4) Bottaro, S.; Bussi, G.; Pinamonti, G.; Reisser, S.; Boomsma, W.; Lindorff-Larsen, K. Barnaba: Software for Analysis of Nucleic Acids Structures and Trajectories. RNA 2019, 25, 219-231.

(5) Cornell, W. D.; Cieplak, P.; Bayly, C. I.; Gould, I. R.; Merz, K. M.; Ferguson, D. M.; Spellmeyer, D. C.; Fox, T.; Caldwell, J. W.; Kollman, P. A. A Second Generation Force Field for the Simulation of Proteins, Nucleic Acids, and Organic Molecules. J. Am. Chem. Soc. 1995, 117, 5179-5197.

(6) Pérez, A.; Marchán, I.; Svozil, D.; Sponer, J.; Cheatham, T. E.; Laughton, C. A.; Orozco, M. Refinement of the AMBER Force Field for Nucleic Acids: Improving the Description of $\alpha / \gamma$ Conformers. Biophys $J$ 2007, 92, 3817-3829.

(7) Zgarbová, M.; Otyepka, M.; Šponer, J.; Mládek, A.; Banáš, P.; Cheatham, T. E.; Jurečka, P. Refinement of the Cornell et al. Nucleic Acids Force Field Based on Reference Quantum Chemical Calculations of Glycosidic Torsion Profiles. J. Chem. Theory Comput. 2011, 7, 2886-2902. 
(8) Steinbrecher, T.; Latzer, J.; Case, D. A. Revised AMBER Parameters for Bioorganic Phosphates. J. Chem. Theory Comput. 2012, 8, 4405-4412.

(9) Izadi, S.; Anandakrishnan, R.; Onufriev, A. V. Building Water Models: A Different Approach. J. Phys. Chem. Lett. 2014, 5, 3863-3871.

(10) Kumar, S.; Bouzida, D.; Swendsen, R. H.; Kollman, P. A.; Rosenberg, J. M. The Weighted Histogram Analysis Method for Free-energy Calculations on Biomolecules. I: The Method. J. Comput. Chem. 1992, 13, 1011-1021.

(11) Branduardi, D.; Bussi, G.; Parrinello, M. Metadynamics with adaptive Gaussians. J. Chem. Theory Comput. 2012, 8, 2247-2254.

(12) Norgaard, A. B.; Ferkinghoff-Borg, J.; Lindorff-Larsen, K. Experimental parameterization of an energy function for the simulation of unfolded proteins. Biophys. J. 2008, 94, 182-192. 